\title{
Transplacental Passage and Subcellular Accumulation of Lanthanum within Maternal and Fetus side of Placenta: A Transmission Electron Microscopy Study
}

\author{
Badri Nedra ${ }^{1 *}$, Mhamdi Maroua ${ }^{1}$, Florea Adrian ${ }^{2}$, Horea Matei ${ }^{2}$, Tekaya Walid-Habib ${ }^{3}$, Hila Lamia ${ }^{4}$ Maghraoui Samira ${ }^{1}$ and Tekaya Leila ${ }^{1}$ \\ ${ }^{1}$ Laboratry of Physiology, Faculty of Medicine of Tunis (Université de Tunis EL Manar). 15 Rue Djebel Lakhdhar. 1007, La rabta. Tunis-Tunisie \\ ${ }^{2}$ Department of Cell and Molecular Biology, University of Medicine and Pharmacy « luliu Hatieganu » 6 L. Pasteur St, Cluj-Napoca 400349 Cluj Napoca, Romania. \\ ${ }^{3}$ Department of Stomatology, Faculty of Dentistry of Monastir (Université de Monastir-Monastir), Tunisie. \\ ${ }^{4}$ Department of Genetics, Faculty of Medicine of Tunis (Université de Tunis EL Manar). 15 Rue Djebel Lakhdhar. 1007, La rabta. Tunis-Tunisie.
}

"Corresponding author: Badri Nedra, Laboratry of Physiology, Faculty of Medicine of Tunis (Universite de Tunis EL Manar). 15 Rue Djebel Lakhdhar. 1007, La rabta. Tunis-Tunisie., Tel: 00216-24987400; E-mail:

nedra.badri@yahoo.fr

Received date: May 18, 2018; Accepted date: May 30, 2018; Published date: June 05, 2018

Copyright: (c) 2018 Nedra B et al. This is an open-access article distributed under the terms of the Creative Commons Attribution License, which permits unrestricted use, distribution, and reproduction in any medium, provided the original author and source are credited.

\begin{abstract}
Placenta is a privileged organ which has a storage function during pregnancy. Thus, it establishes an interface between maternal circulation and fetus. Therefore, any deviation from normal placental development may constitute a potential threat to fetus growth. In this work, we try to study the subcellular behavior as well as the effects of lanthanum (La), on placenta of gestating rats after its intraperitoneal injection under soluble solution. Using TEM, the ultrastructural observations of placenta tissues from lanthanum-treated rats showed many electron-dense surcharges in the lysosomes of maternal connective tissues from maternal part of placenta as well as syncytiotrophoblast and cytotrophoblast from fetus side of placenta respectively. At a concentration of $48 \mathrm{mg} / \mathrm{kg}$, lanthanum was found to cause change in nucleus contouring, vacuolations, expansion of the rough endoplasmic reticulum, mitochondrial sufferance and cytoplasmic rarefication. These modifications covered both side of placenta. The ultrastructural study of control rats showed a normal aspect of the ultrastructure of the two parts of placenta. Finally, our data strongly suggests the transplacental passage of lanthanum and its accumulation within the lysosomes of both side of placenta which may constitute a potential threat to fetus growth and development. We try through this work to study the cellular distribution of lanthanum after its intraperitoneal administration as a soluble solution to attempt to characterize its eventual transfer across the placenta from mother to the fetus.
\end{abstract}

Keywords: Pregnancy; Placenta; Syncytiotrophoblast; Cytotrophoblast; Lanthanum; Lysosome; Transmission Electron Microscopy (TEM)

\section{Introduction}

The placenta grows rapidly and exhibits marked changes in morphological structure according to fetal development. Although the placenta is a temporary organ, which delivers all-necessary nutrients to the fetus, as well as a barrier to prevent passage of toxic substances, including metals, which may interfere with placental function at many levels. Therefore, any deviation from normal placental development has adverse effects on the maintenance of pregnancy as well as fetal growth and development [1].

Lanthanum (La), an element which belongs to the group of the rare earth elements. Hence, its most common biologic ligands are carboxyl and phosphate groups $\left(\mathrm{PO}_{4}{ }^{3-}\right)$ with which it can form very tight, insoluble complexes. Because of its important physical and chemical effects, this rare earth has been widely used in pharmacological and electronic industries. Thus, it has antibacterial effects and regulates cellular immunity. It also been proposed that lanthanum is a calciumand aluminum-free phosphate binder that can be used for the treatment of the hyperphosphatemia of chronic renal failure [2]. In industry, lanthanum is used in camera and telescope lenses and compounds containing lanthanum are used extensively in carbon lighting applications, such as studio lighting and cinema projection. In addition, compounds of lanthanum could stimulate the growth of agricultural products, hence, they have been widely used in farms as an important trace fertilizer [3]. We try through this work to study the cellular distribution of lanthanum after its intraperitoneal administration as a soluble solution to attempt to characterize its eventual transfer across the placenta from mother to the fetus.

\section{Methods}

\section{Rats}

Sixteen virgin females with an average body weight of 140-150 g and eight fertile rats of Wistar strain were obtained from the Animal Experimentation Unit of the Medical School of Tunis (Tunisia) and used for experimentation.

Rats were housed in polystyrene cages which were adequate for accommodating three rats each (ratio of 1 male: 2 females to ensure gestation), under controlled conditions of temperature $\left(27^{\circ} \mathrm{C}\right)$, humidity (75\%) and light (12 h light/dark cycle).

To detect pregnancy from the first day, vaginal smears were carried out daily. The selected pregnant rats were next randomly allocated in two equal groups: 
The first group (G1) is made up of 8 pregnant rats received since the $16^{\text {th }}$ day of gestation a soluble solution of lanthanum (Sigma-Aldrich) as four chronic intraperitoneal injections of $12 \mathrm{mgl}^{-1} \mathrm{ml}$ each. At the end of experimentation each rat accumulated a total dose of $48 \mathrm{mg}$ of lanthanum/Kg of body weight.

The second group (G2) consisting of 8 pregnant rats, was used as a control. These animals received a placebo $(\mathrm{NaCl}$ at $9 \% ; 154 \mathrm{mmol} / \mathrm{L} \mathrm{Na}$ +; $285 \mathrm{mOsm} / \mathrm{L}$ Sigma-Aldrich), under the same experimental conditions.

$24 \mathrm{~h}$ after the last injection (day 20), all rats were anesthetized, sacrificed and fragments of placenta were removed from the fetus of control and treated groups.

All procedures involving animal care and experimental procedures were performed according to the approved animal care protocols of the Ethics Committee on Animal Welfare in accordance with the international principles for the use of animals in Toxicology.

\section{Tissue Preparation}

Tissue were prepared according to standard techniques of TEM, the obtained fragments of placenta were cut in to $1 \mathrm{~mm}^{3}$ in volume and immediately fixed by immersion at $4^{\circ} \mathrm{C}$ for 2 days in Glutaraldehyde $3 \%$ solution (Sigma-Aldrich). Two successive washing of the fragments were performed in $0.5 \mathrm{M}$ cacodylate buffer and a post fixation with osmium tetroxide at $1 \%$ (Sigma-Aldrich) was released, for 2 days. At $37^{\circ}$, cuts were next dehydrated in graded concentrations of ethyl alcohol, bathed twice in propylene oxide, and finally embedded in epoxy resin. The samples were incubated for $48 \mathrm{~h}$ at $45^{\circ} \mathrm{C}$ and for $24 \mathrm{~h}$ at $60^{\circ} \mathrm{C}$ for complete resin polymerization. Semi-thin sections of 100 to $150 \mathrm{~nm}$ thicknesses were obtained with a Bromma 8800 Ultratome III (LKB, Sweden) to evaluate the general aspect of placental tissues. The tissue territories of the selected semi-thin sections were cut into ultrathin sections of $70 \mathrm{~nm}$ of thickness, collected on 300-mesh copper grids and finally contrasted in two steps with uranyl acetate and lead citrate in order to increase the contrast of specific structures of the cells and tissues.

\section{Brightfield Microscopy}

In order to select the fields of interest, semithin sections were stained with a mixture of blue borax methylene and blue toluidine solutions $(1 \mathrm{~V} / 1 \mathrm{~V})$ and finally deposited on glass slides and observed under a Nikon Eclipse 80i (Nikon, Tokyo, Japan) light microscope.

\section{Transmission Electron Microscopy (TEM)}

TEM is a very powerful tool for material science. A high energy beam of electrons is shone through a very thin sample. Samples must be prepared according to a specific protocol which allowed them to retain the cellular ultrastructure and eventually gave the possibility to obtain ultrathin sections that let the electron beam pass through. Colorations with heavy metals are also possible to increase the contrast of specific structures of the samples previously placed on observation checklists. Ultrastructural observations were carried out using a Jeol JEM1010 (Jeol, Tokyo, Japan). The following operating conditions were used: accelerating voltage $80 \mathrm{Kv}$ and various magnification powers.

\section{Results}

\section{Maternal side of placenta}

The ultrastructural observations of maternal side of placenta of pregnant lanthanum-treated rats identified the presence of many electron-dense surcharges within the lysosomes of maternal connective tissues. Some of these lysosomes have lost their membrane. Changes in the architectural histology of the tissue translated by swollen mitochondria were in addition noticed (Figure 1). Control placenta samples didn't reveal any surcharge or histological modifications (Figure 2).

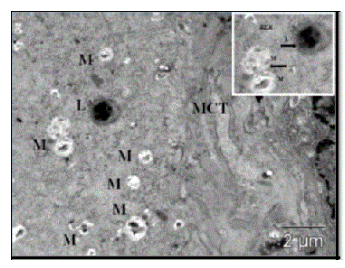

Figure 1: (Magnification X 10.000) A micrograph of maternal connective tissue (MCT). Showed many swollen mitochondria (M) with no visible cristae, charged lysosome (L) without membrane were observed.

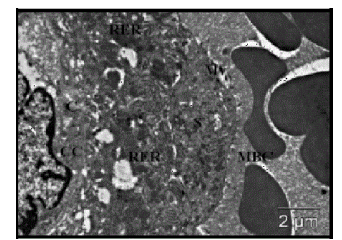

Figure 2: (Magnification X 10.000) Normal ultrastructural aspects of placenta of control rats. TEM showed maternal blood capillary (MBC), syncitiotrophoblast (S) with their microvilli (Mv), Cytotrophoblast (C) with their characteristic cells (CC). Nucleus $(\mathrm{N})$ and rough endoplasmic reticulum (RER) were indeed noticed.

\section{Fetus side of placenta}

\section{Syncytiotrophoblast}

Ultrastructural study of syncytiotrophoblast tissue, from lanthanum-treated rats, allowed the observation of loaded down lysosomes charged with electron dense deposits within the cytoplasm of this variety of tissue. Outside the cells, the low amount of extracellular matrix also leaved empty spaces conferring the aspect of extracellular vacuolations associated to suffering mitochondria were also seen (Figure 3). However our experimental investigations didn't reveal any intralysosomal inclusions in the various territories of placenta from pregnant rats which receive saline solution under the same experimental conditions (Figure 2). 


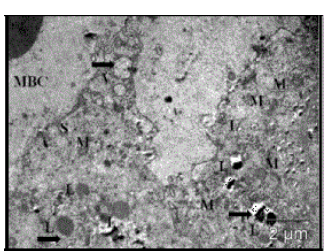

Figure 3: (Magnification X 10.000) Ultrastructural study of syncitiotrophoblast (S) from pregnant lanthanum treated rats. TEM allowed to locate the presence of loaded down lysosomes (L) with different sizes, extensive intercellular vacuolization $(\mathrm{V})$, altered mitochondria $(\mathrm{M})$, a portion of maternal blood capilary $(\mathrm{MBC})$.

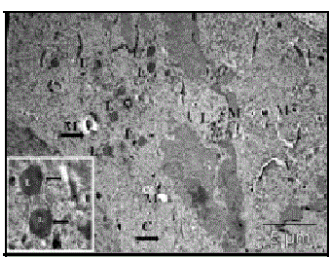

Figure 4: (Magnification X 5000) Micrograph of a portion of cytotrophoblast (C) showed:many loaded down lysosomes (L) without their membrane and swollen mitochondria $(\mathrm{M})$.

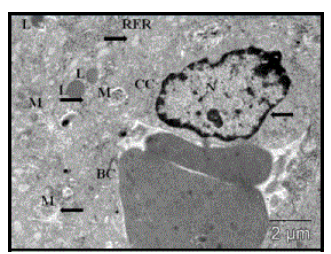

Figure 5: (Magnification X 10.000) Ultrastructural micrograph of cytotrophoblast from lanthanum-treated rats showed cytotrophoblast cells (CC) with euchromatic, polymorphous nuclei $(\mathrm{N})$. In the cytoplasm, mitochondria $(\mathrm{M})$ were altered, numerous lysosomes (L) contained electron dense deposits, and dilated rough endoplasmic reticulum (RER), blood capilary (BC) were identified.

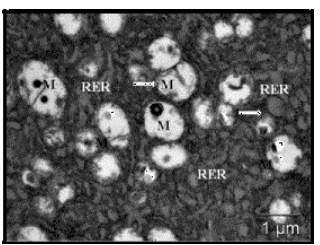

Figure 6: (Magnification X 20.000) Micrograph of a portion of cytotrophoblast from pregnant lanthanum-treated rats showing clearly important degenerative changes translated by swollen mitochondria (M) with electron lucent matrix and no cristae. Marked expansion of rough endoplasmic reticulum (RER) was also highlighted.

\section{Cytotrophoblast}

The ultrastructural study of fetus side of placenta showed clearly the presence of many lysosomes within the cytoplasm, all being charged with an electron-dense material and most of them have lost their membrane. Abnormal profile of mitochondria and polymorphous nuclei were indeed found in some cells (Figures 4 and 5).In another territory of the cytotrophoblast tissue our ultrastructural studies attempting to locate severe mitochondrial suffering with no visible cristas well as rough endoplasmic reticulum profiles were also altered and expanded led to a structural alteration covered those varieties of cells (Figure 6). Neither loaded lysosomes nor histological destruction were detected in placental sections of control rats (Figure 2).

\section{Discussion}

With the application of sensitive techniques of observation and analysis such as X-ray Microanalysis and the Electron Probe Microanalysis, the subcellular behavior of mineral elements within the living organism is well understood. Thus, a phenomenon of selective concentration- precipitation, of these elements within lysosomes of a wide variety of cells was figure out. This process was well elucidated, for the first time in 1974, for some heavy metals, such as gold and uranium, which deposits were detected in proximal renal cells after their subcutaneous administration separately under soluble form to rats [4]. This study allowed the demonstration of a new function of the lysosome [5]. This phenomenon was then generalized on at least 15 elements of Mendeleev's periodic table. For example, the III A group of the Mendeleev chart, such as gallium, which deposits were identified in the lysosomes of tumoral cells [6], and aluminum selectively concentrated in the lysosomes of bone marrow, liver, and mammary gland cells [7-9]. As for rare earths, gadolinium was found in several kinds of cells such as alveolar macrophages, liver cells [10-12], endometrium, myometrium, granulosa and internal theca cells [13]. An intralysosomal detection of cerium was mentionnated in the intestinal mucosa, liver, kidney, suprarenal, and testicular glands [14]. Indeed cerium was pointed out recently within two varities of cells of the uterus (endometrium and myometrium cells) as well as within the granulosa and internal theca cells of the ovary [15]. Moreover, several researches have reported that, after its intraperitoneal administration, lanthanum, the mineral of choice in this work, have a great affinity to the lysosomes of alveolar macrophages, bone marrow and spleen $[16,17]$, as well as hepatocytes and Kupffer cells $[14,18]$. In addition our recent published data dealing with the concentration of lanthanum within the female reproductive organs confirmed the presence of intralysosomal electron-dense deposits of this rare earth in the lysosomes of both endometrial and myometrial cells of the uterus as well as in ovarian cells such as granulosa cells of mature follicles and internal theca cells [15]. Administered orally, lanthanum deposits were found in the lysosomes of duodenal enterocytes $[14,18,19]$.

Despite these reported researches dealing with the distribution of mineral elements and rare earths in the organism none of them have concerned the investigation of intracellular accumulation of other elements such as rare earths; lanthanum, within the placenta. A privileged organ which has a storage function, during pregnancy and its importance in the biology of reproduction, in the maintenance of pregnancy as well as fetal growth and development.

This work is the first paper detailed a transplacental passage and a subcellular accumulation of lanthanum within the lysosomes of maternal and fetus side of placenta. Thus, after four intraperitoneal 
administrations of a soluble solution of lanthanum to pregnant Wistar rats, our ultrastructural observations showed that the injected element was sequestered within the lysosomes of maternal connective tissues from maternal part of placenta as well as syncytiotrophoblast and cytotrophoblast from fetus side of placenta respectively as electrondense deposits. Indeed, lanthanum with used dose triggered intracellular damage, covered both side of placenta especially fetus side. These modifications were translated by change in nucleus contouring, vacuolations, expansion of the rough endoplasmic reticulum, mitochondrial sufferance and cytoplasmic rarefication. No electrons dense deposits were observed in placental sections from control rats.

We speculated that these observed deposits are composed of lanthanum and phosphorus, but that direct demonstration was not possible by conventional transmission electron microscopy. On the other hand, we compared the images obtained from treated group versus the control ones in which the absence of charged lysosomes was noticed which confirms the presence of lanthanum within the lysosomes of the different cellular varieties of treated rats. Indeed, by analogy with previous studies, using the Electron Probe Microanalysis and the Ion Mass Spectrometry allowed determining the chemical composition of the intralysosomal deposits, we can said that the deposits found within the lysosomes of each variety of cell were probably formed of lanthanum associated each time with phosphorus. These observations are in accordance with those reported by Hocine et al. as well as by Manoubi et al. for the same element demonstrating that this rare earth was accumulated within the lysosomes of alveolar macrophages, bone marrow and spleen $[16,17]$, as well as hepatocytes and Kupffer cells $[14,18]$. In addition, our data strongly agree with those reported earlier by us, for the same element, which deposits were located respectively in endometrium, myometrium, granulosa and theca interna cells after its intraperitoneal administration [15].

Our ultrastructural results reminder many previous studies describing that cerium, a mineral element which belonging to the same group of lanthanum, was found within the lysosomes of intestinal mucosa, liver, kidney, suprarenal, and testicular glands cells [14] as well as endometrium and myometrium cells, granulosa and internal theca cells of the ovary [15]. In addition, our observed deposits were similar to those founded with gadolinium in alveolar macrophages [17,10], liver cells, spleen, bone marrow, proximal renal cells [20], endometrium, myometrium, granulosa and internal theca cells [13]. Indeed, the same processes of precipitation within the lysosomes were highlighted with heavy metals. For example, lead was found to cross the placental barrier and was found to easily accumulate in the placental tissues [21]. Also mercury surcharge was found on primary trophoblast $[22,23]$. It is well established that maternal exposure to heavy metals may cause placental damage and change the transportation of essential trace metals to the fetus. Available studies showed that cadmium accumulated in the placental tissues and in high levels of exposure could alter endocrine function, causing various reproductive problems and changes in the cells may be the cause of the death of the fetus by placental dysfunction or failure [24]. Although our results presented common highlights with these previous studies, in fact during our experiments we found that there were cases of death respectively of the mother and fetus, congenital malformations were observed in the fetus, weight loss and mother's abortions were also noticed. All these results confirmed that lanthanum across trough the placental barrier from the mother to the fetus demonstrating that the placental barrier is not completely impermeable to the passage of harmful substances. Although in an unpublished data relative to very recent study based into spectrometric analyzes using the ICP-AES technique, we were able to detect lanthanum with 170,71-372,17 ppm in placental tissues of treated rats as well as in fetus tissue with a concentration of $11,59-85,17 \mathrm{ppm}$.

\section{Conclusion}

Our data demonstrated that lanthanum, a rare earth, was probably selectively concentrated under insoluble form within the lysosomes of fetal side of placenta, as well as within the lysosomes of maternal side of placenta. As result of the failure of lysosomes in the sequestration of the total amount of lanthanum, visible changes in tissue and cells involving cytoplasmic vacuolization, marked expansion of the rough endoplasmic reticulum, mitochondrial damages, irregular nuclear outline and enlarged perinuclear space of cells covered both side of placenta. Also our experimental results reported cases of death, malformations, weight loss and abortions allowed as to conclude that there was a transplacental passage of the administered element so a placental barrier failure.

\section{References}

1. Furukawa SH, Usuda S, Abe K, Hagio M, Ogawa I. (2011) Toxicological pathology in the rat placenta. J Toxicol Pathol 24: 95-111.

2. Bernard L, Anthony L, Daniel A, Nadya R, Natalie M, et al. (2005) Chronic renal failure is associated with increase tissue depletion of lanthanum after 28 day oral administration. Kidney International 67: 1062-1069.

3. Guo BS, Zhu WM, Xiong BK, Ji Liu Z, Wu ZM (1990) Rare Earths in Agriculture China.Agricultural Science and Technology Press, Beijing. Pages: 11

4. Galle P (1974) Role of lysosomes and mitochondria in the phenomena of concentration and elimination of mineral elements (uranium and gold) by the kidney. J Microsc 19:17-33.

5. DE Duve C, Wattiaux R (1966) Functions of lysosomes. Annu Rev Physiol 28:435-492.

6. Berry JP, Escaig F, Poupon MP, Galle P (1983) Localization of gallium in tumor cells. Electron microscopy, electron probe microanalysis and analytical ion microscopy. Int J Nucl Med Biol 10:199-204.

7. Wartin A, Galle P (1986) Sites of aluminum accumulation in bone marrow: study using electron microscopy, ionic microscopy and $\mathrm{X}$ ray microanalysis. Biol Cell 57:63-75.

8. Galle P, Campos H, Chadenas D (1987) Abnormal concentration of aluminum in lysosomes of a parathyroid primitive adenoma. Ann Pathol 7: 65-68.

9. Ayadi A, EL Hili A, Galle P, Tekaya L (2008) Role of lysosomes of duodenal and hepatic cells in the gadolinium accumulation phenomenon. C R Biologies 331: 357-362.

10. Hocine N, Berry JP, Jaafoura MH, Escaig F, et al. (1995) Subcellular localization of gadolinium injected as soluble salt in rats: a microanalytical study. Cell Mol Biol 41: 271-278.

11. Ayadi A, EL Hili A, Tekaya L (2008) Lysosomes of the liver and the duodenal enterocytes, a site of handling of two rare earths. Ultrastructural and microanalytical study. Biol Trace Elem Res 124:40-51.

12. Ahlem A, Samira M, Ali EH, Pierre G, Leila T (2008) Ultrastructural study of the intracellular behaviour of four mineral elements in the lactating mammary gland cells. Microsc Res Tech 71:849-855.

13. Mhamdi M, Florea A, Badri N, Maghraoui S, Matei H, et al. (2017).The role of lysosomes in the phenomenon of concentration of aluminum and indium in the female reproductive system. An ultrastructural study. J Trace Elem Med Biol 44:59-64.

14. Manoubi L, Hocine N, Jaafoura H, EL Hili A, Galle P (1998) Subcellular localization of cerium in intestinal mucosa, liver, kidney, suprarenal and 
Citation: Badri Nedra (2018) Transplacental Passage and Subcellular Accumulation of Lanthanum within Maternal and Fetus side of Placenta: A Transmission Electron Microscopy Study. Biol Syst Open Access 7: 186. doi:10.4172/2329-6577.1000186

Page 5 of 5

testicle glands, after cerium administration in the rat. J Trace Microprobe Tech 16:209-219.

15. Badri N, Florea A, Mhamdi M, Matei H, Tekaya WH, et al. (2017)Toxicological effects and ultrastructural changes induced by lanthanum and cerium in ovary and uterus of Wistar rats. J Trace Elem Med Biol 44:349-355.

16. Hocine N, Berry JP, Escaig F, Galle P (1994) Mapping and localization of gadolinium. A study using ion microanalysis and electron microprobe. Les éditions de Physique, pp. 841-842.

17. Hocine N, Berry JP, Jaafoura MH, Galle P (1994)Intracellular localization of gadolinium, lanthanum and terbium. Three rare earths. A microanalytical study using ion microanalysis and electron microprobe, Mandal Ion in Biology and Medicine, Editions of Physics, pages: 193-198.

18. Manoubi-Tekaya L, Hocine N, Galle P (2000) Roles of lysosomes in the intracellular behaviour of mineral elements: case of lanthanum. Tunis Med 78:195-200.

19. Florent Ch, Tekaya L, Escaig F, Labejof L, Mouthon G, et al. (2001)Analytical microscopy observations of rat enterocytes after oral administration of soluble salts of lanthanides, actinides and elements of group III-A of the periodic chart. Cell Mol Biol 47:419-425.

20. Berry JP (1996) The role of lysosomes in the selective concentration of mineral elements. A microanalytical study. Cell Mol Biol 42: 395-411.

21. Robert S, Grzegorz F, Magdalena C, Katarzyna C, Anna KK, et al. (2011) Accumulation of cadmium and lead in placenta and amnion of women from upper silesian region Poland. J Env Res Develop 5: 871-879.

22. Ni Z, Mao Q. ATP-binding cassette efflux transporters in human placenta (2011) Curr Pharm Biotechnol 12: 674-685.

23. Meyer zu Schwabedissen HE, Jedlitschky G, Gratz M, Haenisch S, Linnemann K,et al. (2005)Variable expression of MRP2 (ABCC2) in human placenta: influence of gestational age and cellular differentiation. Drug Metab Dispos 33: 896-904.

24. Zakrzewska M, Biaonska D, Sawicka Kapusta K (2002) Cadmium accumulation in fetus and placenta of bank voles. (Clethrionomys glareolus, Schreber 1780) Bull Environ Contam Toxicol 69: 829-834. 